\title{
SOBOLEV AND HARDY-LITTLEWOOD-SOBOLEV INEQUALITIES: DUALITY AND FAST DIFFUSION
}

\author{
Jean Dolbeault
}

\begin{abstract}
In the euclidean space of dimension $d \geq 3$, Sobolev and Hardy-LittlewoodSobolev inequalities can be related by duality. We investigate how to relate these inequalities using the flow of a fast diffusion equation. Up to a term which is needed for homogeneity reasons, the difference of the two terms in Sobolev's inequality can be seen as the derivative with respect to time along the flow of an entropy functional based on the Hardy-Littlewood-Sobolev inequality. A similar result also holds in dimension $d=2$ with Sobolev and Hardy-Littlewood-Sobolev inequalities replaced, respectively, by a variant of Onofri's inequality and by the logarithmic Hardy-Littlewood-Sobolev inequality, while the flow is determined by a super-fast diffusion equation.

By considering second derivatives in time of the entropy functional along the flow of the fast diffusion equation, we obtain an improvement of Sobolev's inequality in terms of the entropy. However, for integrability reasons, the method is restricted to $d \geq 5$.
\end{abstract}

\section{Introduction}

In dimension $d \geq 3$, it is well known since Lieb's paper [24] that Hardy-LittlewoodSobolev inequalities are dual of Sobolev's inequalities. We will investigate this duality using the flow of a fast diffusion equation which has been considered in [16]. In a recent paper, [9], Carlen et al noticed that Hardy-Littlewood-Sobolev inequalities in dimension $d \geq 3$ and some special Gagliardo-Nirenberg inequalities can be related through another fast diffusion equation. Although Carlen et al [9] have motivated the present paper (see Remark 2.1), the two approaches are so far unrelated.

Consider Sobolev's inequality in $\mathbb{R}^{d}, d \geq 3$,

$$
\|u\|_{\mathrm{L}^{2^{*}\left(\mathbb{R}^{d}\right)}}^{2} \leq \mathrm{S}_{d}\|\nabla u\|_{\mathrm{L}^{2}\left(\mathbb{R}^{d}\right)}^{2}, \quad \forall u \in \mathcal{D}^{1,2}\left(\mathbb{R}^{d}\right),
$$

where $S_{d}$ is the Aubin-Talenti constant (see $[1,30]$ and also $[5,28]$ for earlier related results) and $2^{*}=\frac{2 d}{d-2}$. The space $\mathcal{D}^{1,2}\left(\mathbb{R}^{d}\right)$ is defined as the completion of smooth solutions with compact support w.r.t. the norm $w \mapsto\|w\|:=\left(\|\nabla w\|_{\mathrm{L}^{2}\left(\mathbb{R}^{d}\right)}^{2}+\right.$ $\left.\|w\|_{\mathrm{L}^{2^{*}}\left(\mathbb{R}^{d}\right)}^{2}\right)^{1 / 2}$. The Hardy-Littlewood-Sobolev inequality

$$
\mathrm{S}_{d}\|v\|_{\mathrm{L}^{\frac{2 d}{d+2}}\left(\mathbb{R}^{d}\right)}^{2} \geq \int_{\mathbb{R}^{d}} v(-\Delta)^{-1} v d x \quad \forall v \in \mathrm{L}^{\frac{2 d}{d+2}}\left(\mathbb{R}^{d}\right)
$$

involves the same optimal constant, $\mathrm{S}_{d}$.

Received by the editors January 12, 2012.

Key words and phrases. Sobolev spaces; Hardy-Littlewood-Sobolev inequality; logarithmic Hardy-Littlewood-Sobolev inequality; Sobolev's inequality; Onofri's inequality; GagliardoNirenberg inequality; extremal functions; duality; best constants; stereographic projection; fast diffusion equation; extinction. - AMS classification (2010). 26D10; 46E35; 35K55. 
To a convex functional $F$, we may associate the functional $F^{*}$ defined by Legendre's duality as $F^{*}[v]:=\sup \left(\int_{\mathbb{R}^{d}} u v d x-F[u]\right)$. For instance, to $F_{1}[u]=\frac{1}{2}\|u\|_{\mathrm{L}^{p}\left(\mathbb{R}^{d}\right)}^{2}$ defined on $\mathrm{L}^{p}\left(\mathbb{R}^{d}\right)$, we henceforth associate $F_{1}^{*}[v]=\frac{1}{2}\|v\|_{\mathrm{L}^{q}\left(\mathbb{R}^{d}\right)}^{2}$ on $\mathrm{L}^{q}\left(\mathbb{R}^{d}\right)$ where $p$ and $q$ are Hölder conjugate exponents: $1 / p+1 / q=1$. The supremum can be taken for instance on all functions in $\mathrm{L}^{p}\left(\mathbb{R}^{d}\right)$, or, by density, on the smaller space of the functions $u \in \mathrm{L}^{p}\left(\mathbb{R}^{d}\right)$ such that $\nabla u \in \mathrm{L}^{2}\left(\mathbb{R}^{d}\right)$. Similarly, to $F_{2}[u]=\frac{1}{2} \mathrm{~S}_{d}\|\nabla u\|_{\mathrm{L}^{2}\left(\mathbb{R}^{d}\right)}^{2}$, we associate $F_{2}^{*}[v]=\frac{1}{2} \mathrm{~S}_{d}^{-1} \int_{\mathbb{R}^{d}} v(-\Delta)^{-1} v d x$ where $(-\Delta)^{-1} v=G_{d} * v$ with $G_{d}(x)=$ $\frac{1}{d-2}\left|\mathbb{S}^{d-1}\right|^{-1}|x|^{2-d}$. As a straightforward consequence of Legendre's duality, if we have a functional inequality of the form $F_{1}[u] \leq F_{2}[u]$, then we have the dual inequality $F_{1}^{*}[v] \geq F_{2}^{*}[v]$. In this sense, (1.1) and (1.2) are dual of each other, as it has been noticed in [24].

In the present paper, we investigate this duality using a nonlinear diffusion equation. If $v$ is a positive solution of the following fast diffusion equation:

$$
\frac{\partial v}{\partial t}=\Delta v^{m} \quad t>0, \quad x \in \mathbb{R}^{d}
$$

and if we define $\mathrm{H}(t):=\mathrm{H}_{d}[v(t, \cdot)]$, with

$$
\mathrm{H}_{d}[v]:=\int_{\mathbb{R}^{d}} v(-\Delta)^{-1} v d x-\mathrm{S}_{d}\|v\|_{\mathrm{L}^{\frac{2 d}{d+2}}\left(\mathbb{R}^{d}\right)}^{2},
$$

then we observe that

$$
\frac{1}{2} \mathrm{H}^{\prime}=-\int_{\mathbb{R}^{d}} v^{m+1} d x+\mathrm{S}_{d}\left(\int_{\mathbb{R}^{d}} v^{\frac{2 d}{d+2}} d x\right)^{\frac{2}{d}} \int_{\mathbb{R}^{d}} \nabla v^{m} \cdot \nabla v^{\frac{d-2}{d+2}} d x,
$$

where $v=v(t, \cdot)$ is a solution of (1.3). With the choice $m=\frac{d-2}{d+2}$, we find that $m+1=\frac{2 d}{d+2}$, so that the above identity can be rewritten with $u=v^{m}$ as follows.

Proposition 1.1. Assume that $d \geq 3$ and $m=\frac{d-2}{d+2}$. If $v$ is a solution of (1.3) with nonnegative initial datum in $\mathrm{L}^{2 d /(d+2)}\left(\mathbb{R}^{d}\right)$, then

$$
\begin{aligned}
& \frac{1}{2} \frac{d}{d t}\left[\int_{\mathbb{R}^{d}} v(-\Delta)^{-1} v d x-\mathrm{S}_{d}\|v\|_{\mathrm{L}^{\frac{2 d}{d+2}}\left(\mathbb{R}^{d}\right)}^{2}\right] \\
& \quad=\left(\int_{\mathbb{R}^{d}} v^{m+1} d x\right)^{\frac{2}{d}}\left[\mathrm{~S}_{d}\|\nabla u\|_{\mathrm{L}^{2}\left(\mathbb{R}^{d}\right)}^{2}-\|u\|_{\mathrm{L}^{2^{*}}\left(\mathbb{R}^{d}\right)}^{2}\right] \geq 0
\end{aligned}
$$

As a consequence, one can prove that (1.2), which amounts to $\mathrm{H} \leq 0$, is a consequence of (1.1), that is $\mathrm{H}^{\prime} \geq 0$, by showing that $\lim _{\sup _{t>0}} \mathrm{H}(t)=0$. In this way, we also recover the property that $u=v^{m}$ is an optimal function for (1.1) if $v$ is optimal for (1.2).

As we shall see next, $t \mapsto \mathrm{H}(t)$ is concave function. Using this property, we can actually obtain optimal integral remainder terms which improve on the usual Sobolev inequality (1.1), but only when $d \geq 5$, for integrability reasons: see Theorem 2.1. A slightly weaker result is the following improved Sobolev inequality, which relates (1.1) and (1.2). 
Theorem 1.1. Assume that $d \geq 5$ and let $q=\frac{d+2}{d-2}$. There exists a positive constant $\mathcal{C}$ such that, for any $w \in \mathcal{D}^{1,2}\left(\mathbb{R}^{d}\right)$, we have

$$
\begin{aligned}
& \mathrm{S}_{d}\left\|w^{q}\right\|_{\mathrm{L}^{\frac{2 d}{d+2}}\left(\mathbb{R}^{d}\right)}^{2}-\int_{\mathbb{R}^{d}} w^{q}(-\Delta)^{-1} w^{q} d x \\
& \quad \leq \mathcal{C}\|w\|_{\mathrm{L}^{2^{*}}\left(\mathbb{R}^{d}\right)}^{\frac{8}{d-2}}\left[\|\nabla w\|_{\mathrm{L}^{2}\left(\mathbb{R}^{d}\right)}^{2}-\mathrm{S}_{d}\|w\|_{\mathrm{L}^{2^{*}}\left(\mathbb{R}^{d}\right)}^{2}\right] .
\end{aligned}
$$

Moreover, we know that $\mathcal{C} \leq\left(1+\frac{2}{d}\right)\left(1-e^{-d / 2}\right) \mathrm{S}_{d}$.

The issue of adding correction terms to Sobolev's inequality and getting improved versions of it has been studied in [4], in the bounded domain case. Also see [7] for an earlier related paper. However, in [4], Brezis and Lieb were also asking the question of measuring the distance to the manifold of optimal functions in the case of the euclidean space. A few years later, Bianchi and Egnell gave an answer in [6] using compactness methods, with no explicit value of the constant. Since then, considerable efforts have been devoted to obtain quantitative improvements of Sobolev's inequality. On the whole euclidean space, nice results based on rearrangements have been obtained in [13] and we refer to [12] for an interesting review of the various results that have been established over the years. It has to be noted that they are all of different nature than the inequality in Theorem 1.1, which is the main result of this paper.

Our approach is based on the evolution equation (1.3). Many papers have been devoted to the study of the asymptotic behaviour of the solutions, in bounded domains: [3, 19, 29], or in the whole space: [20, 22, 27]. In particular, the CauchySchwarz inequality has been repeatedly used, for instance in [3, 29], and turns out to be a key tool in the proof of Theorem 1.1, as well as the solution with separation of variables, which is related to the Aubin-Talenti optimal function for (1.1). See Section 2 for details. The novelty in our approach is to consider the problem from the point of view of the functional associated to (1.2) using (1.3) with $m=(d-2) /(d+2)$.

\section{Improved Sobolev inequalities}

This section is devoted to the proof of Theorem 1.1. We shall assume that

$$
m=\frac{d-2}{d+2} \quad \text { and } \quad d \geq 3 .
$$

From the computations of Section 1, it is clear that the maximum of $\mathrm{H}_{d}[v]$ is achieved if and only if $u=v^{m}$ is an extremal for Sobolev's inequality. This is of course consistent with the fact that extremal points for Hardy-Littlewood-Sobolev inequalities and Sobolev inequalities are related through Legendre's duality precisely by the relation $u=v^{m}$.

It is also straightforward to check that (1.3) admits special solutions with separation of variables such that, for any $T>0$,

$$
\bar{v}_{T}(t, x)=c(T-t)^{\alpha}(F(x))^{\frac{d+2}{d-2}}, \quad \forall(t, x) \in(0, T) \times \mathbb{R}^{d},
$$

where $\alpha=(d+2) / 4, c^{1-m}=4 m d, m=\frac{d-2}{d+2}, p=d /(d-2)$ and $F$ is the AubinTalenti solution of $-\Delta F=d(d-2) F^{(d+2) /(d-2)}$. Such a solution vanishes at $t=T$ and this behaviour is generic in a large class of solutions. Define

$$
\|v\|_{*}:=\sup _{x \in \mathbb{R}^{d}}\left(1+|x|^{2}\right)^{d+2}|v(x)| \text {. }
$$


Lemma 2.1. [16, 31]. For any solution $v$ of (1.3) with nonnegative, not identically zero initial datum $v_{0} \in \mathrm{L}^{2 d /(d+2)}\left(\mathbb{R}^{d}\right)$, there exists $T>0, \lambda>0$ and $x_{0} \in \mathbb{R}^{d}$ such that $v(t, \cdot) \not \equiv$ for any $t \in(0, T)$ and

$$
\lim _{t \rightarrow T_{-}}(T-t)^{-\frac{1}{1-m}}\|v(t, \cdot) / \bar{v}(t, \cdot)-1\|_{*}=0
$$

with $\bar{v}(t, x)=\lambda^{(d+2) / 2} \bar{v}_{T}\left(t,\left(x-x_{0}\right) / \lambda\right)$.

We shall refer to such a solution as a solution vanishing at time $T$. The above result has been established first in [16] when $\left\|v_{0}\right\|_{*}$ is finite and extended to solutions corresponding to any initial data $v_{0} \in \mathrm{L}_{+}^{2 d /(d+2)}\left(\mathbb{R}^{d}\right)$ in $[31$, Theorem 7.10]. In this framework, it is easy to establish further a priori estimates as follows.

Lemma 2.2. Let $d \geq 3$ and $m=(d-2) /(d+2)$. If $v$ is a solution of $(1.3)$ vanishing at time $T>0$ with initial datum $v_{0} \in \mathrm{L}_{+}^{2 d /(d+2)}\left(\mathbb{R}^{d}\right)$, then for any $t \in(0, T)$ we have the estimates

$$
\begin{aligned}
& \left(\frac{4(T-t)}{(d+2) \mathrm{S}_{d}}\right)^{\frac{d}{2}} \leq \int_{\mathbb{R}^{d}} v^{m+1}(t, x) d x \leq \int_{\mathbb{R}^{d}} v_{0}^{m+1} d x, \\
& \left\|\nabla v^{m}(t, \cdot)\right\|_{\mathrm{L}^{2}\left(\mathbb{R}^{d}\right)}^{2} \geq \mathrm{S}_{d}^{-1}\left(\frac{4(T-t)}{d+2}\right)^{\frac{d}{2}-1}
\end{aligned}
$$

and the vanishing time $T$ is bounded by

$$
T \leq \frac{1}{4}(d+2) \mathrm{S}_{d}\left(\int_{\mathbb{R}^{d}} v_{0}^{m+1} d x\right)^{\frac{2}{d}} .
$$

If additionally $d \geq 5$, then $T \geq \frac{d+2}{2 d} \int_{\mathbb{R}^{d}} v_{0}^{m+1} d x\left\|\nabla v_{0}^{m}\right\|_{\mathrm{L}^{2}\left(\mathbb{R}^{d}\right)}^{-2}$ and

$$
\begin{aligned}
\int_{\mathbb{R}^{d}} v^{m+1}(t, x) d x & \geq \int_{\mathbb{R}^{d}} v_{0}^{m+1} d x-\frac{2 d}{d+2} t\left\|\nabla v_{0}^{m}\right\|_{\mathrm{L}^{2}\left(\mathbb{R}^{d}\right)}^{2}, \\
\left\|\nabla v^{m}(t, \cdot)\right\|_{\mathrm{L}^{2}\left(\mathbb{R}^{d}\right)}^{2} & \leq\left\|\nabla v_{0}^{m}\right\|_{\mathrm{L}^{2}\left(\mathbb{R}^{d}\right)}^{2} .
\end{aligned}
$$

Proof. By definition of the vanishing time, we find that $\mathrm{J}(t):=\int_{\mathbb{R}^{d}} v(t, x)^{m+1} d x$ satisfies $\mathrm{J}(t)>0$ for any $t \in(0, T), \mathrm{J}(T)=0$ and, using the equation and integrating by parts,

If $d \geq 5$, then we also have

$$
\mathrm{J}^{\prime}=-(m+1)\left\|\nabla v^{m}\right\|_{\mathrm{L}^{2}\left(\mathbb{R}^{d}\right)}^{2} \leq-\frac{m+1}{\mathrm{~S}_{d}} \mathrm{~J}^{1-\frac{2}{d}} .
$$

$$
\mathrm{J}^{\prime \prime}=2 m(m+1) \int_{\mathbb{R}^{d}} v^{m-1}\left(\Delta v^{m}\right)^{2} d x \geq 0 .
$$

It is easy to check that such an estimate makes sense if $v=\bar{v}_{T}$. For a general solution, this is also true as can be seen by rewriting the problem on $\mathbb{S}^{d}$ as in [16]. It is then clear that integrability conditions for $v$ are exactly the same as for $\bar{v}_{T}$.

By integrating the first inequality from $t$ to $T$, we find that

$$
-\mathrm{J}(t)^{\frac{2}{d}}=\mathrm{J}(T)^{\frac{2}{d}}-\mathrm{J}(t)^{\frac{2}{d}} \leq-\frac{4(T-t)}{(d+2) \mathrm{S}_{d}} \quad \forall t \in[0, T)
$$

which gives an upper bound for $T$ by choosing $t=0$. The second inequality shows the decay of $t \mapsto\left\|\nabla v^{m}(t, \cdot)\right\|_{\mathrm{L}^{2}\left(\mathbb{R}^{d}\right)}^{2}$ and gives the estimate $0=\mathrm{J}(T) \geq \mathrm{J}(0)+T \mathrm{~J}^{\prime}(0)$, thus providing a lower bound for $T$. 
For later purpose, let us notice that

$$
\frac{\mathrm{J}^{\prime}}{\mathrm{J}} \leq-\frac{m+1}{\mathrm{~S}_{d}} \mathrm{~J}^{-\frac{2}{d}} \leq-\kappa \quad \text { with } \quad \kappa:=\frac{2 d}{d+2} \frac{1}{\mathrm{~S}_{d}}\left(\int_{\mathbb{R}^{d}} v_{0}^{m+1} d x\right)^{-\frac{2}{d}} \leq \frac{d}{2 T} .
$$

Next, we are going to compute the second derivative of $\mathbf{H}(t)=\mathbf{H}_{d}[v(t, \cdot)]$ w.r.t. $t$ along the flow of (1.3). For this purpose, we assume that $d \geq 5$ (see Remark 2.2 below) and notice that by the Cauchy-Schwarz inequality, we have

$$
\begin{aligned}
\left\|\nabla v^{m}\right\|_{\mathrm{L}^{2}\left(\mathbb{R}^{d}\right)}^{4} & =\left(\int_{\mathbb{R}^{d}} v^{(m-1) / 2} \Delta v^{m} \cdot v^{(m+1) / 2} d x\right)^{2} \\
& \leq \int_{\mathbb{R}^{d}} v^{m-1}\left(\Delta v^{m}\right)^{2} d x \int_{\mathbb{R}^{d}} v^{m+1} d x
\end{aligned}
$$

As a consequence, we get that $\mathrm{Q}(t):=\left\|\nabla v^{m}(t, \cdot)\right\|_{\mathrm{L}^{2}\left(\mathbb{R}^{d}\right)}^{2}\left(\int_{\mathbb{R}^{d}} v^{m+1}(t, x) d x\right)^{-(d-2) / d}$ is monotone decreasing. More precisely, with $m=(d-2) /(d+2)$,

$$
\Lambda(t):=\frac{\int_{\mathbb{R}^{d}}\left|\nabla(v(t, x))^{m}\right|^{2} d x}{\int_{\mathbb{R}^{d}}(v(t, x))^{m+1} d x} \text { and } \mathrm{G}\left(t_{1}, t_{2}\right):=\exp \left[(m+1) \int_{t_{1}}^{t_{2}} \Lambda(s) d s\right],
$$

we get that $\mathrm{Q}^{\prime}=-2 m \mathrm{~J}^{\frac{2}{d}-1} \mathrm{~K}$ with $\mathrm{K}:=\int_{\mathbb{R}^{d}} v^{m-1}\left|\Delta v^{m}+\Lambda v\right|^{2} d x$ and, from

$$
\mathrm{H}^{\prime}=2 \mathrm{~J}\left(\mathrm{~S}_{d} \mathrm{Q}-1\right) \text {, }
$$

we deduce that

$$
\mathrm{H}^{\prime \prime}=\frac{\mathrm{J}^{\prime}}{\mathrm{J}} \mathrm{H}^{\prime}+2 \mathrm{JS}_{d} \mathrm{Q}^{\prime}=-(m+1) \Lambda \mathrm{H}^{\prime}-4 m \mathrm{~S}_{d} \mathrm{~J}^{\frac{2}{d}} \mathrm{~K}
$$

As a consequence, the standard Sobolev inequality can be improved by an integral remainder term.

Theorem 2.1. Assume that $d \geq 5$ and let $q=\frac{d+2}{d-2}$. For any $w \in \mathcal{D}^{1,2}\left(\mathbb{R}^{d}\right)$ such that $\left\|w^{q}\right\|_{*}<\infty$, we have

$$
\begin{gathered}
\mathrm{S}_{d}\left\|w^{q}\right\|_{\mathrm{L}^{\frac{2 d}{d+2}}\left(\mathbb{R}^{d}\right)}^{2}-\int_{\mathbb{R}^{d}} w^{q}(-\Delta)^{-1} w^{q} d x+\frac{4}{q} \mathrm{~S}_{d} \int_{0}^{T} d t \int_{0}^{t} \mathrm{~J}^{\frac{2}{d}}(s) \mathrm{K}(s) \mathrm{G}(t, s) d s \\
=2\|w\|_{\mathrm{L}^{2^{*}}\left(\mathbb{R}^{d}\right)}^{\frac{4}{d-2}}\left[\|\nabla w\|_{\mathrm{L}^{2}\left(\mathbb{R}^{d}\right)}^{2}-\mathrm{S}_{d}\|w\|_{\mathrm{L}^{2^{*}}\left(\mathbb{R}^{d}\right)}^{2}\right] \int_{0}^{T} \mathrm{G}(t, 0) d t .
\end{gathered}
$$

Here $v$ is the solution of $(1.3)$ with $v_{0}=w^{q}, m=(d-2) /(d+2)=1 / q, T$ is the vanishing time, $\Lambda$ and $G$ are defined by $(2.2)$, and we recall that $\mathrm{J}(t)=\int_{\mathbb{R}^{d}} v^{m+1}(t, x) d x$, $\mathrm{K}(t)=\int_{\mathbb{R}^{d}} v^{m-1}(t, x)\left|\Delta v^{m}(t, x)+\Lambda(t) v(t, x)\right|^{2} d x$.

Proof. The identity follows from (2.3) after an integration from 0 to $t$ and another one from 0 to $T$. Details are left to the reader.

Recall that the standard Hardy-Littlewood-Sobolev inequality (1.2) amounts to

$$
\mathrm{S}_{d}\left\|w^{q}\right\|_{\mathrm{L}^{\frac{2 d}{d+2}}\left(\mathbb{R}^{d}\right)}^{2}-\int_{\mathbb{R}^{d}} w^{q}(-\Delta)^{-1} w^{q} d x=-\mathrm{H}_{d}\left[w^{q}\right] \geq 0 .
$$

The main drawback of Theorem 2.1 is that several quantities can be computed only through the evolution equation and are therefore nonexplicit. With simple estimates, we can however get rid of such quantities. This is the purpose of Theorem 1.1. 
Proof of Theorem 1.1. Notice that $\left\|w^{q}\right\|_{\mathrm{L}^{2 d /(d+2)}\left(\mathbb{R}^{d}\right)}^{2}=\|w\|_{\mathrm{L}^{2^{*}\left(\mathbb{R}^{d}\right)}}^{1+2 / d}$, so that the inequality holds in the space $\mathcal{D}^{1,2}\left(\mathbb{R}^{d}\right)$. Theorem 1.1 can be established first in the setting of smooth functions such that $\left\|w^{q}\right\|_{*}<\infty$ and then arguing by density.

From (2.1) and (2.3), we know that

$$
\mathrm{H}^{\prime \prime} \leq-\kappa \mathrm{H}^{\prime} \quad \text { with } \quad \kappa=\frac{2 d}{d+2} \frac{1}{\mathrm{~S}_{d}}\left(\int_{\mathbb{R}^{d}} v_{0}^{m+1} d x\right)^{-2 / d} .
$$

By writing that $-\mathrm{H}(0)=\mathrm{H}(T)-\mathrm{H}(0) \leq \mathrm{H}^{\prime}(0)\left(1-e^{-\kappa T}\right) / \kappa$ and using the estimate $\kappa T \leq d / 2$, we obtain the result.

Remark 2.1. In [9], Carlen et al noticed that Hardy-Littlewood-Sobolev inequalities and Gagliardo-Nirenberg inequalities can be related through the fast diffusion equation (1.3) with exponent $m=d /(d+2)$, when $d \geq 3$. The key computation goes as follows:

$$
\begin{aligned}
\frac{1}{2} \frac{d}{d t} \mathrm{H}_{d}[v(t, \cdot)] & =\frac{1}{2} \frac{d}{d t}\left[\int_{\mathbb{R}^{d}} v(-\Delta)^{-1} v d x-\mathrm{S}_{d}\|v\|_{\mathrm{L}^{\frac{2 d}{d+2}}\left(\mathbb{R}^{d}\right)}^{2}\right] \\
& =\frac{d(d-2)}{(d-1)^{2}} \mathrm{~S}_{d}\|u\|_{\mathrm{L}^{q+1}\left(\mathbb{R}^{d}\right)}^{4 /(d-1)}\|\nabla u\|_{\mathrm{L}^{2}\left(\mathbb{R}^{d}\right)}^{2}-\|u\|_{\mathrm{L}^{2 q}\left(\mathbb{R}^{d}\right)}^{2 q}
\end{aligned}
$$

with $u=v^{(d-1) /(d+2)}$ and $q=(d+1) /(d-1)$, thus proving that $\frac{d}{d t} \mathrm{H}_{d}[v(t, \cdot)]$ has a sign because of Gagliardo-Nirenberg inequalities: see Remark 3.1 below. According, e.g., to [15], the asymptotic behaviour of (1.3) with $m=\frac{d}{d+2}$ is governed by the Barenblatt self-similar solutions. By integrating along the flow of (1.3), this provides an integral remainder term, which improves on Hardy-Littlewood-Sobolev inequalities (1.2).

Remark 2.2. The reader may wonder why the condition $d \geq 5$ has been introduced in the setting of Theorems 1.1 and 2.1, while it was not present in Proposition 1.1. This has been done for integrability reasons. As can easily be checked, $\int_{\mathbb{R}^{d}} v^{m-1}\left(\Delta v^{m}\right)^{2} d x$ does not make sense if $v$ is a solution with separation of variables and if $d=3$ or 4 . Since the solution $v$ of (1.3) uniformly converges to a solution with separation of variables, $\bar{v}$ with the notations of Lemma 2.1, as well as the derivatives of $v$ converge to the derivatives of $\bar{v}$ (as follows by parabolic regularity theory), the computation of $\mathrm{H}^{\prime \prime}$ cannot be carried out unless $d \geq 5$.

This does not mean that one cannot extend the method to the cases $d=3$ or $d=4$, by considering functionals relative to $\bar{v}$, but such a question has not been studied yet. We shall see examples of relative functionals (in dimension $d=2$ ) in the next section.

\section{The two-dimensional case}

3.1. Logarithmic Hardy-Littlewood-Sobolev and Onofri inequalities. In dimension $d=2$, Onofri's inequality [26]

$$
\log \left(\int_{\mathbb{R}^{2}} e^{g} d \mu\right)-\int_{\mathbb{R}^{2}} g d \mu \leq \frac{1}{16 \pi} \int_{\mathbb{R}^{2}}|\nabla g|^{2} d x, \quad \forall g \in \mathcal{D}\left(\mathbb{R}^{d}\right)
$$


plays the role of Sobolev's inequality in higher dimensions. Here the probability measure $d \mu$ is defined by

$$
d \mu(x):=\mu(x) d x \quad \text { with } \quad \mu(x):=\frac{1}{\pi\left(1+|x|^{2}\right)^{2}}, \quad \forall x \in \mathbb{R}^{2} .
$$

Although there are many ways to derive such an inequality, it is interesting to see it as a limit case of some Gagliardo-Nirenberg inequalities which are deeply connected with the fast diffusion equation. Details will be provided in Section 3.2.

The logarithmic Hardy-Littlewood-Sobolev inequality can be written as follows: for any $f \in \mathrm{L}_{+}^{1}\left(\mathbb{R}^{2}\right)$ with $M=\int_{\mathbb{R}^{2}} f d x$, such that $f \log f,\left(1+\log |x|^{2}\right) f \in \mathrm{L}^{1}\left(\mathbb{R}^{2}\right)$, we have

$$
\int_{\mathbb{R}^{2}} f \log \left(\frac{f}{M}\right) d x+\frac{2}{M} \int_{\mathbb{R}^{2} \times \mathbb{R}^{2}} f(x) f(y) \log |x-y| d x d y+M(1+\log \pi) \geq 0
$$

This inequality has recently attracted lots of attention in connection with the KellerSegel model or in geometry: see for instance $[8,18,25]$. It is also known to be a limit case of the Hardy-Littlewood-Sobolev inequalities in dimension $d=2$, as was noted for instance in [11], and can also be characterized as the dual inequality of Onofri's inequality in dimension $d=2$. Details will be given in Section 3.3.

Our purpose is to relate (3.1) to (3.2) by the mean of a nonlinear flow. This will be done in Section 3.4, in a limit case of the fast diffusion equation, involving a logarithmic nonlinearity. We will obtain the counterpart of Proposition 1.1 in dimension $d=2$. The analogue of Theorem 1.1 is for the moment still unknown.

3.2. From Gagliardo-Nirenberg inequalities to Onofri's inequality. Consider the following sub-family of Gagliardo-Nirenberg inequalities

$$
\|f\|_{\mathrm{L}^{2 p}\left(\mathbb{R}^{d}\right)} \leq \mathrm{C}_{p, d}\|\nabla f\|_{\mathrm{L}^{2}\left(\mathbb{R}^{d}\right)}^{\theta}\|f\|_{\mathrm{L}^{p+1}\left(\mathbb{R}^{d}\right)}^{1-\theta}
$$

with $\theta=\theta(p):=\frac{p-1}{p} \frac{d}{d+2-p(d-2)}, 1<p \leq \frac{d}{d-2}$ if $d \geq 3$ and $1<p<\infty$ if $d=2$. Such an inequality holds for any smooth function $f$ with sufficient decay at infinity and, by density, for any function $f \in \mathrm{L}^{p+1}\left(\mathbb{R}^{d}\right)$ such that $\nabla f$ is square integrable. We shall assume that $C_{p, d}$ is the best possible constant. In [15], it has been established that equality holds in (3.3) if $f=F_{p}$ with

$$
F_{p}(x)=\left(1+|x|^{2}\right)^{-\frac{1}{p-1}}, \quad \forall x \in \mathbb{R}^{d}
$$

and that all extremal functions are equal to $F_{p}$ up to a multiplication by a constant, a translation and a scaling. If $d \geq 3$, the limit case $p=d /(d-2)$ corresponds to Sobolev's inequality and one recovers the results of Aubin and Talenti in [1, 30], with $\theta=1$ : the optimal functions for (1.1) are, up to scalings, translations and multiplications by a constant, all equal to $F_{d /(d-2)}(x)=\left(1+|x|^{2}\right)^{-(d-2) / 2}$, and

$$
\mathrm{S}_{d}=\left(\mathrm{C}_{d /(d-2), d}\right)^{2} \text {. }
$$

Remark 3.1. Consider again the setting of Remark 2.2. An explicit computation shows that $\frac{d(d-2)}{(d-1)^{2}} \mathrm{~S}_{d}=\left(\mathrm{C}_{q, d}\right)^{2 q}$, thus proving that optimality is achieved simultaneously in both sides of (2.4), for any $d \geq 3$. As observed in [9], a similar property holds 
in dimension $d=2$ : using the flow of (1.3) with exponent $m=1 / 2$, it turns out that

$$
\begin{aligned}
& \frac{\|v\|_{\mathrm{L}^{1}\left(\mathbb{R}^{2}\right)}}{8} \frac{d}{d t}\left[\frac{4 \pi}{\|v\|_{\mathrm{L}^{1}\left(\mathbb{R}^{2}\right)}} \int_{\mathbb{R}^{2}} v(-\Delta)^{-1} v d x-\int_{\mathbb{R}^{2}} v \log v d x\right] \\
& =\|u\|_{\mathrm{L}^{4}\left(\mathbb{R}^{2}\right)}^{4}\|\nabla u\|_{\mathrm{L}^{2}\left(\mathbb{R}^{2}\right)}^{2}-\pi\|v\|_{\mathrm{L}^{6}\left(\mathbb{R}^{2}\right)}^{6},
\end{aligned}
$$

which is the Gagliardo-Nirenberg inequality (3.3) with $d=2, q=3$, and an optimal constant such that $\pi\left(\mathrm{C}_{3,2}\right)^{6}=1$.

When $p \rightarrow 1$, the inequality becomes an equality, so that we may differentiate both sides with respect to $p$ and recover the euclidean logarithmic Sobolev inequality in optimal scale invariant form (see [15, 21, 33] for details).

We now investigate the limit $p \rightarrow \infty$ in (3.3) when $d=2$ : Onofri's inequality (3.1) can be obtained in the limit. As an endpoint case of the family of the GagliardoNirenberg inequalities (3.3), it plays the role of Sobolev's inequality in dimension $d \geq 3$.

Proposition 3.1. Assume that $g \in \mathcal{D}\left(\mathbb{R}^{d}\right)$ is such that $\int_{\mathbb{R}^{2}} g d \mu=0$ and let

$$
f_{p}:=F_{p}\left(1+\frac{g}{2 p}\right)
$$

where $F_{p}$ is defined by (3.4). Then we have

$$
1 \leq \lim _{p \rightarrow \infty} C_{p, 2} \frac{\left\|\nabla f_{p}\right\|_{L^{2}\left(\mathbb{R}^{2}\right)}^{\theta(p)}\left\|f_{p}\right\|_{L^{p+1}\left(\mathbb{R}^{2}\right)}^{1-\theta(p)}}{\left\|f_{p}\right\|_{L^{2 p}\left(\mathbb{R}^{2}\right)}}=\frac{e^{\frac{1}{16 \pi} \int_{\mathbb{R}^{2}}|\nabla g|^{2} d x}}{\int_{\mathbb{R}^{2}} e^{g} d \mu} .
$$

We recall that $\mu(x):=\frac{1}{\pi}\left(1+|x|^{2}\right)^{-2}$, and $d \mu(x):=\mu(x) d x$. Such a result of approximation is not surprising in view of the existing literature (see for instance [2] in the case of interpolations inequalities on the sphere or [17] in which Onofri's inequality is seen as a limit case of the Caffarelli-Kohn-Nirenberg inequalities). Moreover this result nicely complements [15].

Proof. We can rewrite (3.3) as

$$
\frac{\int_{\mathbb{R}^{2}}|f|^{2 p} d x}{\int_{\mathbb{R}^{2}}\left|F_{p}\right|^{2 p} d x} \leq\left(\frac{\int_{\mathbb{R}^{2}}|\nabla f|^{2} d x}{\int_{\mathbb{R}^{2}}\left|\nabla F_{p}\right|^{2} d x}\right)^{\frac{p-1}{2}} \frac{\int_{\mathbb{R}^{2}}|f|^{p+1} d x}{\int_{\mathbb{R}^{2}}\left|F_{p}\right|^{p+1} d x}
$$

and observe that, with $f=f_{p}$, we have:

(i) $\lim _{p \rightarrow \infty} \int_{\mathbb{R}^{2}}\left|F_{p}\right|^{2 p} d x=\int_{\mathbb{R}^{2}} \frac{1}{\left(1+|x|^{2}\right)^{2}} d x=\pi$ and

$$
\lim _{p \rightarrow \infty} \int_{\mathbb{R}^{2}}\left|f_{p}\right|^{2 p} d x=\int_{\mathbb{R}^{2}} F_{p}^{2 p}\left(1+\frac{g}{2 p}\right)^{2 p} d x=\int_{\mathbb{R}^{2}} \frac{e^{g}}{\left(1+|x|^{2}\right)^{2}} d x
$$

so that $\int_{\mathbb{R}^{2}}\left|f_{p}\right|^{2 p} d x / \int_{\mathbb{R}^{2}}\left|F_{p}\right|^{2 p} d x$ converges to $\int_{\mathbb{R}^{2}} e^{g} d \mu$ as $p \rightarrow \infty$,

(ii) $\int_{\mathbb{R}^{2}}\left|F_{p}\right|^{p+1} d x=(p-1) \pi / 2, \lim _{p \rightarrow \infty} \int_{\mathbb{R}^{2}}\left|f_{p}\right|^{p+1} d x=\infty$, but

$$
\lim _{p \rightarrow \infty} \frac{\int_{\mathbb{R}^{2}}\left|f_{p}\right|^{p+1} d x}{\int_{\mathbb{R}^{2}}\left|F_{p}\right|^{p+1} d x}=1
$$


(iii) expanding the square and integrating by parts, we find that

$$
\begin{aligned}
\int_{\mathbb{R}^{2}}\left|\nabla f_{p}\right|^{2} d x & =\frac{1}{4 p^{2}} \int_{\mathbb{R}^{2}} F_{p}^{2}|\nabla g|^{2} d x-\int_{\mathbb{R}^{2}}\left(1+\frac{g}{2 p}\right)^{2} F_{p} \Delta F_{p} d x \\
& =\frac{1}{4 p^{2}} \int_{\mathbb{R}^{2}}|\nabla g|^{2} d x+\frac{2 \pi}{p+1}+o\left(p^{-2}\right) .
\end{aligned}
$$

Here we have used $\int_{\mathbb{R}^{2}}\left|\nabla F_{p}\right|^{2} d x=\frac{2 \pi}{p+1}$ and the condition $\int_{\mathbb{R}^{2}} g d \mu=0$ in order to discard one additional term of the order of $p^{-2}$. On the other hand, we find that

$$
\left(\frac{\int_{\mathbb{R}^{2}}\left|\nabla f_{p}\right|^{2} d x}{\int_{\mathbb{R}^{2}}\left|\nabla F_{p}\right|^{2} d x}\right)^{\frac{p-1}{2}} \sim\left(1+\frac{p+1}{8 \pi p^{2}} \int_{\mathbb{R}^{2}}|\nabla g|^{2} d x\right)^{\frac{p-1}{2}} \sim e^{\frac{1}{16 \pi} \int_{\mathbb{R}^{2}}|\nabla g|^{2} d x}
$$

as $p \rightarrow \infty$. Collecting these estimates concludes the proof.

3.3. Legendre duality. Now we state the duality which relates Onofri's inequality (3.1) and the logarithmic Hardy-Littlewood-Sobolev inequality (3.2) in $\mathbb{R}^{2}$. With

$$
F_{1}[u]:=\log \left(\int_{\mathbb{R}^{2}} e^{u} d \mu\right) \text { and } F_{2}[u]:=\frac{1}{16 \pi} \int_{\mathbb{R}^{2}}|\nabla u|^{2} d x+\int_{\mathbb{R}^{2}} u \mu d x,
$$

Onofri's inequality amounts to $F_{1}[u] \leq F_{2}[u]$.

Proposition 3.2. For any $v \in \mathrm{L}_{+}^{1}\left(\mathbb{R}^{2}\right)$ with $\int_{\mathbb{R}^{2}} v d x=1$, such that $v \log v$ and $\left(1+\log |x|^{2}\right) v \in \mathrm{L}^{1}\left(\mathbb{R}^{2}\right)$, we have

$$
F_{1}^{*}[v]-F_{2}^{*}[v]=\int_{\mathbb{R}^{2}} v \log \left(\frac{v}{\mu}\right) d x-4 \pi \int_{\mathbb{R}^{2}}(v-\mu)(-\Delta)^{-1}(v-\mu) d x \geq 0 .
$$

This result has already been observed by several authors: see for instance [2, 10, 11] for the duality argument on the two-dimensional sphere, which gives the above inequality on $\mathbb{R}^{2}$ by the stereographic projection. For completeness, let us give a proof directly in the euclidean setting. We essentially follow the computation of [8, Appendix] but explicitly compute the constants.

Proof. On the Orlicz space of functions $u \in \mathrm{L}_{\text {loc }}^{1}\left(\mathbb{R}^{2}\right)$ such that $\nabla u \in \mathrm{L}^{2}\left(\mathbb{R}^{2}\right)$ and $e^{u} \in \mathrm{L}^{1}\left(\mathbb{R}^{2}, d \mu\right)$, Legendre's transform gives

$$
\begin{aligned}
& F_{1}^{*}[v]=\int_{\mathbb{R}^{2}} u v d x-\log \left(\int_{\mathbb{R}^{2}} e^{u} d \mu\right) \quad \text { with } \quad \log v=\log \mu+u-\log \left(\int_{\mathbb{R}^{2}} e^{u} d \mu\right), \\
& F_{2}^{*}[v]=\int_{\mathbb{R}^{2}} u(v-\mu) d x-\frac{1}{16 \pi} \int_{\mathbb{R}^{2}}|\nabla u|^{2} d x \quad \text { with } \quad-\Delta u=8 \pi(v-\mu),
\end{aligned}
$$

which proves the result.

A useful observation is the fact that $-\Delta \log \mu=8 \pi \mu$ can be inverted as

$$
(-\Delta)^{-1} \mu=\frac{1}{8 \pi} \log \mu+C .
$$

It is then easy to check that $u:=(-\Delta)^{-1} \mu=G_{2} * \mu$, with $G_{2}(x)=-\frac{1}{2 \pi} \log |x|$, is such that $u(0)=0$, which determines $C=\frac{1}{8 \pi} \log \pi$. Hence with the notations of 
Proposition 3.2, we may observe that

$$
\begin{aligned}
& F_{1}^{*}[v]=\int_{\mathbb{R}^{2}} v \log \left(\frac{v}{\mu}\right) d x \\
& F_{2}^{*}[v]=\pi \int_{\mathbb{R}^{2}} v(-\Delta)^{-1} v d x-\int_{\mathbb{R}^{2}} v \log \mu d x-1-\log \pi .
\end{aligned}
$$

Collecting these observations, we find that

$$
F_{1}^{*}[v]-F_{2}^{*}[v]=\int_{\mathbb{R}^{2}} v \log v d x-4 \pi \int_{\mathbb{R}^{2}} v(-\Delta)^{-1} v d x+1+\log \pi \geq 0 .
$$

For any $f \in \mathrm{L}_{+}^{1}\left(\mathbb{R}^{2}\right)$ with $M=\int_{\mathbb{R}^{2}} f d x$, such that $f \log f,\left(1+\log |x|^{2}\right) f \in \mathrm{L}^{1}\left(\mathbb{R}^{2}\right)$, this inequality, written for $v=f / M$, is nothing else than the logarithmic HardyLittlewood-Sobolev inequality (3.1).

3.4. Duality through the flow of a fast diffusion equation. We may now proceed in the case $d=2$ as we $\operatorname{did}$ for $d \geq 3$. Let

$$
\mathrm{H}_{2}[v]:=\int_{\mathbb{R}^{2}}(v-\mu)(-\Delta)^{-1}(v-\mu) d x-\frac{1}{4 \pi} \int_{\mathbb{R}^{2}} v \log \left(\frac{v}{\mu}\right) d x .
$$

Assume that $v$ is a positive solution of

$$
\frac{\partial v}{\partial t}=\Delta \log \left(\frac{v}{\mu}\right) \quad t>0, \quad x \in \mathbb{R}^{2}
$$

which replaces (1.3). Then we have the analogue of Proposition 1.1.

Proposition 3.3. Assume that $d=2$. If $v$ is a solution of (3.5) with nonnegative initial datum $v_{0}$ in $\mathrm{L}^{1}\left(\mathbb{R}^{2}\right)$ such that $\int_{\mathbb{R}^{2}} v_{0} d x=1$, $v_{0} \log v_{0} \in \mathrm{L}^{1}\left(\mathbb{R}^{2}\right)$ and $v_{0} \log \mu \in$ $\mathrm{L}^{1}\left(\mathbb{R}^{2}\right)$, then

$$
\frac{d}{d t} \mathrm{H}_{2}[v(t, \cdot)]=\frac{1}{16 \pi} \int_{\mathbb{R}^{2}}|\nabla u|^{2} d x-\int_{\mathbb{R}^{2}}\left(e^{\frac{u}{2}}-1\right) u d \mu
$$

with $\log (v / \mu)=u / 2$.

The r.h.s. in the above identity is not exactly the nonnegative functional which can be deduced from Onofri's inequality (3.1), but both are related by an inequality, which goes beyond a simple applications of Jensen's inequality. If $d \nu=e^{u / 2} d \mu$ is a probability measure, using the convexity of $u \mapsto u e^{u / 2}$, we may indeed observe that

$$
\log \left(\int_{\mathbb{R}^{2}} e^{u} d \mu\right)=\log \left(\int_{\mathbb{R}^{d}} e^{\frac{u}{2}} d \nu\right) \geq \int_{\mathbb{R}^{d}} \frac{u}{2} d \nu=\frac{1}{2} \int_{\mathbb{R}^{2}} e^{\frac{u}{2}} d \mu .
$$

What we need to prove is that the inequality holds without the $\frac{1}{2}$ factor. This follows from the fact that the function

$$
h(t):=\log \left(\int_{\mathbb{R}^{2}} e^{t u} d \mu\right)
$$

is such that $h(0)=h(1 / 2)=0$, that $h$ is convex, and that $h^{\prime \prime}$ is nondecreasing. A proof will be given in the Section 3.5. 
Lemma 3.1. For any smooth function $u$ on $\mathbb{R}^{2}$ such that $\int_{\mathbb{R}^{2}} e^{u / 2} d \mu=1$, we have

$$
\log \left(\int_{\mathbb{R}^{2}} e^{u} d \mu\right) \geq \int_{\mathbb{R}^{2}} e^{\frac{u}{2}} d \mu .
$$

In Proposition 3.3, the right-hand side is therefore nonnegative by Onofri's inequality:

$$
\frac{d}{d t} \mathrm{H}_{2}[v(t, \cdot)] \geq \frac{1}{16 \pi} \int_{\mathbb{R}^{2}}|\nabla u|^{2} d x+\int_{\mathbb{R}^{2}} u d \mu-\log \left(\int_{\mathbb{R}^{2}} e^{u} d \mu\right) \geq 0 .
$$

As in [9], an integration w.r.t. $t$ provides an improved inequality with an integral remainder term. As a consequence of Lemma 3.1 and of Onofri's inequality (3.1), we also have the following inequality.

Corollary 3.1. For any $u \in \mathcal{D}\left(\mathbb{R}^{2}\right)$ such that $\int_{\mathbb{R}^{2}} e^{u / 2} d \mu=1$, we have

$$
\frac{1}{16 \pi} \int_{\mathbb{R}^{2}}|\nabla u|^{2} d x \geq \int_{\mathbb{R}^{2}}\left(e^{\frac{u}{2}}-1\right) u d \mu .
$$

This inequality can of course be extended by density to the natural Orlicz space for which all integrals are well defined.

Notice that, in (3.6), we only obtain an inequality, while Proposition 1.1 was providing an identity. A natural candidate to replace the functional J of Section 2 (see in particular Lemma 2.2) in dimension $d \geq 3$ is, in dimension $d=2$, the functional $\mathrm{J}:=2 \int_{\mathbb{R}^{2}} v(\log (v / \mu)-1) d x$. A quick computation shows that, for homogeneity considerations, there is no analogue of the Cauchy-Schwarz inequality which was the key ingredient in the proof of Theorem 1.1.

3.5. Proofs. The computation of $\frac{d}{d t} \mathrm{H}_{2}[v(t, \cdot)]$ in Section 3.4 is formal but can easily be justified after noticing that the image $w$ of $v$ by the inverse stereographic projection on the sphere $\mathbb{S}^{2}$, up to a scaling, solves the equation

$$
\frac{\partial w}{\partial t}=\Delta_{\mathbb{S}^{2}} \log w
$$

More precisely, if $x=\left(x_{1}, x_{2}\right) \in \mathbb{R}^{2}$, then $u$ and $w$ are related by

$$
w(t, y)=\frac{u(t, x)}{4 \pi \mu(x)}, \quad y=\left(\frac{2\left(x_{1}, x_{2}\right)}{1+|x|^{2}}, \frac{1-|x|^{2}}{1+|x|^{2}}\right) \in \mathbb{S}^{2} .
$$

See [31, Section 8.2] for a review of some known results for the logarithmic diffusion equation, or super-fast diffusion equation, $\frac{\partial v}{\partial t}=\Delta \log v$, and $[14,32]$ for earlier results. One of the main features of this equation is that the mass of $v$ is not preserved. However, Equation (3.5) differs through a source term, $\Delta \log \mu$, which compensates for the loss of mass. This is clear from the equation solved by $w$. Another consequence is that the solution of (3.5) globally exists. With these observations in hand, the proof of Proposition 3.3 readily follows. Details are left to the reader.

As already mentioned, mass is preserved along the evolution according of (3.5). For simplicity, we shall assume that $1=\int_{\mathbb{R}^{2}} v_{0} d x=\int_{\mathbb{R}^{2}} v(t, x) d x$ for any $t \geq 0$ and recall that $\int_{\mathbb{R}^{2}} \mu d x=1$.

Proof of Lemma 3.1. Inspired by Latała and Oleszkiewicz [23], let us define

$$
h(t):=\log \left(\int_{\mathbb{R}^{2}} e^{t u} d \mu\right)
$$


Claim 1: $h$ is convex and $h^{\prime}$ is convex. Let us observe that

$$
\left(\int_{\mathbb{R}^{2}} e^{t u} d \mu\right)^{2} h^{\prime \prime}(t)=\int_{\mathbb{R}^{2}} e^{t u} d \mu \int_{\mathbb{R}^{2}} u^{2} e^{t u} d \mu-\left(\int_{\mathbb{R}^{2}} u e^{t u} d \mu\right)^{2} \geq 0
$$

by the Cauchy-Schwarz inequality. With one more derivation, we find that

$$
\begin{aligned}
\left(\int_{\mathbb{R}^{2}} e^{t u} d \mu\right)^{3} h^{\prime \prime \prime}(t)= & \int_{\mathbb{R}^{2}} u^{3} e^{t u} d \mu\left(\int_{\mathbb{R}^{2}} e^{t u} d \mu\right)^{2} \\
& -3 \int_{\mathbb{R}^{2}} u^{2} e^{t u} d \mu \int_{\mathbb{R}^{2}} u e^{t u} d \mu \int_{\mathbb{R}^{2}} e^{t u} d \mu+2\left(\int_{\mathbb{R}^{2}} u e^{t u} d \mu\right)^{3} .
\end{aligned}
$$

Let us prove that $h^{\prime \prime \prime}(t)>0$ for $t \in(0,1)$ a.e. If $\int_{\mathbb{R}^{2}} u e^{t u} d \mu>0$, define

$$
d \nu_{t}:=\frac{e^{t u}}{\int_{\mathbb{R}^{2}} e^{t u} d \mu} d \mu \text { and } v:=\frac{u}{\int_{\mathbb{R}^{2}} u d \nu_{t}},
$$

so that $\int_{\mathbb{R}^{2}} v d \nu_{t}=1$. Then $h^{\prime \prime \prime}(t)$ has the sign of $\int_{\mathbb{R}^{2}}\left(v^{3}-3 v^{2}+2\right) d \nu_{t}$. Taking the constraint into account, a direct optimization shows that an optimal function $v$ takes two values, $1 \pm a$ for some $a>0$ and moreover, $\nu_{t}(\{v=1+a\})=\nu_{t}(\{v=1-a\})$ because of the condition $\int_{\mathbb{R}^{2}} v d \nu_{t}=1$. In such a case, it is straightforward to check that $\int_{\mathbb{R}^{2}}\left(v^{3}-3 v^{2}+2\right) d \nu_{t}=0$, thus proving that $h^{\prime \prime \prime}(t) \geq 0$.

If $\int_{\mathbb{R}^{2}} u e^{t u} d \mu<0$, a similar computation with $v:=-u / \int_{\mathbb{R}^{2}} u d \nu_{t}$ shows that $-h^{\prime \prime \prime}(t)$ has the sign of $\int_{\mathbb{R}^{2}}\left(-v^{3}-3 v^{2}+2\right) d \nu_{t}$ under the condition $\int_{\mathbb{R}^{2}} v d \nu_{t}=-1$, thus proving again that $h^{\prime \prime \prime}(t) \geq 0$.

Since $h^{\prime}(t)=\int_{\mathbb{R}^{2}} u d \nu_{t}$ is monotone increasing, $\int_{\mathbb{R}^{2}} u e^{t u} d \mu=0$ occurs for at most one $t \in[0,1]$ and we can conclude that $h^{\prime}$ is convex.

Claim 2: $h(1) \geq h^{\prime}(1 / 2)$. We know that $h(0)=h(1 / 2)=0$ and, by convexity of $h^{\prime}, h^{\prime \prime}$ is monotone nondecreasing. Let $\kappa:=h^{\prime \prime}(1 / 2)$ and $\mathrm{p}:=h^{\prime}(1 / 2)$. On the one hand, we have $h^{\prime \prime}(t) \leq \kappa$ for any $t \in[0,1 / 2]$, which means that, after integrating from $t<1 / 2$ to $1 / 2$, we have

$$
\mathrm{p}-h^{\prime}(t) \leq \kappa\left(\frac{1}{2}-t\right) \quad \forall t \in\left[0, \frac{1}{2}\right]
$$

Hence, one more integration from 0 to $1 / 2$ gives

$$
\frac{\mathrm{p}}{2}=[\mathrm{p} t-h(t)]_{0}^{1 / 2} \leq\left[\kappa \frac{t}{2}(1-t)\right]_{0}^{1 / 2}=\frac{\kappa}{8} .
$$

On the other hand, we have $h^{\prime \prime}(t) \geq \kappa$ for any $t \in[1 / 2,1]$, which means that, after integrating from $1 / 2$ to $t>1 / 2$, we have

$$
h^{\prime}(t)-\mathrm{p} \geq \kappa\left(t-\frac{1}{2}\right) \quad \forall t \in\left[\frac{1}{2}, 1\right] .
$$

One more integration from $1 / 2$ to 1 gives

$$
h(1)-\frac{\mathrm{p}}{2} \geq\left[\kappa \frac{t}{2}(t-1)\right]_{0}^{1 / 2}=\frac{\kappa}{8}
$$

Collecting the two estimates, we find that $h(1) \geq \mathrm{p}$, which proves the claim. 
Using $h(0)=\log \left(\int_{\mathbb{R}^{2}} d \mu\right)=0$ and $h(1 / 2)=\log \left(\int_{\mathbb{R}^{2}} e^{u / 2} d \mu\right)=0$, we have found that $\mathrm{p}=h^{\prime}(1 / 2)=\int_{\mathbb{R}^{2}} u e^{u / 2} d \mu \leq h(1)=\log \left(\int_{\mathbb{R}^{2}} e^{u} d \mu\right)$. This proves that

$$
\frac{d}{d t} \mathrm{H}_{2}[v(t, \cdot)] \geq \frac{1}{16 \pi} \int_{\mathbb{R}^{2}}|\nabla u|^{2} d x+\int_{\mathbb{R}^{2}} u d \mu-\log \left(\int_{\mathbb{R}^{2}} e^{u} d \mu\right) \geq 0,
$$

where the last inequality is nothing else than Onofri's inequality.

\section{Acknowledgments}

The author thanks J.A. Carrillo and M.J. Esteban for useful suggestions. He also thanks an anonymous referee for relevant comments and suggestions going far beyond the purpose of the present papers. This work has been partially supported by the projects CBDif and EVOL of the French National Research Agency (ANR).

(c) 2010 by the author. This paper may be reproduced, in its entirety, for noncommercial purposes.

\section{References}

[1] T. Aubin, Problèmes isopérimétriques et espaces de Sobolev, J. Differential Geometry 11(4) (1976), 573-598.

[2] W. Beckner, Sharp Sobolev inequalities on the sphere and the Moser-Trudinger inequality, Ann. of Math. (2) 138(1) (1993), 213-242.

[3] J. G. Berryman and C. J. Holland, Stability of the separable solution for fast diffusion, Arch. Rational Mech. Anal. 74(4) (1980), 379-388.

[4] G. Bianchi and H. Egnell, A note on the Sobolev inequality, J. Funct. Anal. 100(1) (1991), $18-24$.

[5] G. Bliss, An integral inequality, J. London Math. Soc. 1(1) (1930), 40.

[6] H. Brezis and E. H. Lieb, Sobolev inequalities with remainder terms, J. Funct. Anal. 62(1) (1985), 73-86.

[7] H. Brezis and L. Nirenberg, Positive solutions of nonlinear elliptic equations involving critical Sobolev exponents, Comm. Pure Appl. Math. 36(4) (1983), 437-477.

[8] V. Calvez and L. Corrias, The parabolic-parabolic Keller-Segel model in $\mathbb{R}^{2}$, Commun. Math. Sci. 6(2) (2008), 417-447.

[9] E. A. Carlen, J. A. Carrillo and M. Loss, Hardy-Littlewood-Sobolev inequalities via fast diffusion flows, Proc. Nat. Acad. Sci. 107(46) (2010), 19696-19701.

[10] E. A. Carlen and M. Loss, Competing symmetries of some functionals arising in mathematical physics, in Stochastic processes, Physics and Geometry (Ascona and Locarno, 1988), 277-288, World Sci. Publ., Teaneck, NJ, 1990.

[11] - Competing symmetries, the logarithmic HLS inequality and Onofri's inequality on $S^{n}$, Geom. Funct. Anal. 2(1) (1992), 90-104.

[12] A. Cianchi, Quantitative Sobolev and Hardy inequalities, and related symmetrization principles, in Sobolev spaces in mathematics. I, Vol. 8 of Int. Math. Ser. (N.Y.), 87-116, Springer, New York (2009).

[13] A. Cianchi, N. Fusco, F. Maggi and A. Pratelli, The sharp Sobolev inequality in quantitative form, J. Eur. Math. Soc. (JEMS) 11(5) (2009), 1105-1139.

[14] P. Daskalopoulos and M. A. del Pino, On a singular diffusion equation, Comm. Anal. Geom. 3(3-4) (1995), 523-542.

[15] M. del Pino and J. Dolbeault, Best constants for Gagliardo-Nirenberg inequalities and applications to nonlinear diffusions, J. Math. Pures Appl. (9) 81(9) (2002), 847-875.

[16] M. del Pino and M. Sáez, On the extinction profile for solutions of $u_{t}=\Delta u^{(N-2) /(N+2)}$, Indiana Univ. Math. J. 50(1) (2001), 611-628.

[17] J. Dolbeault, M. J. Esteban and G. Tarantello, The role of Onofri type inequalities in the symmetry properties of extremals for Caffarelli-Kohn-Nirenberg inequalities, in two space dimensions, Ann. Sc. Norm. Super. Pisa Cl. Sci. (5) 7(2) (2008), 313-341. 
[18] J. Dolbeault and B. Perthame, Optimal critical mass in the two-dimensional Keller-Segel model in $\mathbb{R}^{2}$, C. R. Math. Acad. Sci. Paris 339(9) (2004), 611-616.

[19] V. A. Galaktionov and J. R. King, Fast diffusion equation with critical Sobolev exponent in a ball, Nonlinearity 15(1) (2002), 173-188.

[20] V. A. Galaktionov and L. A. Peletier, Asymptotic behaviour near finite-time extinction for the fast diffusion equation, Arch. Ration. Mech. Anal. 139(1) (1997), 83-98.

[21] L. Gross, Logarithmic Sobolev inequalities, Amer. J. Math. 97(4) (1975), 1061-1083.

[22] J. King, Self-similar behaviour for the equation of fast nonlinear diffusion, Philos. Trans. R. Soc. Lond. Ser. A: Math. Phys. Eng. Sci. 343(1668) (1993), 337.

[23] R. Latała and K. Oleszkiewicz, Between Sobolev and Poincaré, in Geometric aspects of functional analysis, Vol. 1745 of Lecture Notes in Math., 147-168, Springer, Berlin (2000).

[24] E. H. Lieb, Sharp constants in the Hardy-Littlewood-Sobolev and related inequalities, Ann. of Math. (2) 118(2) (1983), 349-374.

[25] K. Okikiolu, Extremals for logarithmic Hardy-Littlewood-Sobolev inequalities on compact manifolds, Geom. Funct. Anal. 17(5) (2008), 1655-1684.

[26] E. Onofri, On the positivity of the effective action in a theory of random surfaces, Comm. Math. Phys. 86(3) (1982), 321-326.

[27] M. A. Peletier and H. F. Zhang, Self-similar solutions of a fast diffusion equation that do not conserve mass, Differential Integral Equations 8(8) (1995), 2045-2064.

[28] G. Rosen, Minimum value for $c$ in the Sobolev inequality $\left\|\phi^{3}\right\| \leq c\|\nabla \phi\|^{3}$, SIAM J. Appl. Math. 21 (1971) 30-32.

[29] G. Savaré and V. Vespri, The asymptotic profile of solutions of a class of doubly nonlinear equations, Nonlinear Anal. 22(12) (1994), 1553-1565.

[30] G. Talenti, Best constant in Sobolev inequality, Ann. Mat. Pura Appl. (4) 110 (1976), 353-372.

[31] J. L. Vázquez, Smoothing and decay estimates for nonlinear diffusion equations, Vol. 33 of Oxford Lecture Series in Mathematics and its Applications, Oxford University Press, Oxford, 2006.

[32] J. L. Vázquez, J. R. Esteban and A. Rodríguez, The fast diffusion equation with logarithmic nonlinearity and the evolution of conformal metrics in the plane, Adv. Differential Equations 1(1) (1996), 21-50.

[33] F. B. Weissler, Logarithmic Sobolev inequalities for the heat-diffusion semigroup, Trans. Amer. Math. Soc. 237 (1978), 255-269.

Ceremade (UmR CNRS 7534), Université Paris-Dauphine, Place de Lattre de Tassigny, 75775 PARIS CÉDEX 16, France

E-mail address: dolbeaul@ceremade.dauphine.fr 